\title{
Una expresión infrecuente de una asociación epidémica desbordada: Tuberculosis y diabetes
}

\author{
Ángela María Giraldo M $^{1}$, Rafael Conde ${ }^{2}$, Hugo Herrera ${ }^{3}$, Jaqueline Mugnier ${ }^{4}$, Carlos A. Torres Duque ${ }^{5}$. \\ 1 Fellow de Neumología, Universidad de la Sabana. Fundación Neumológica Colombiana, Bogotá. \\ 2 Neumólogo, Fundación Neumológica Colombiana, Santa Fe de Bogotá. \\ 3 Médico Patólogo. Fundación Cardioinfantil, Santa Fe de Bogotá. \\ 4 Médica Patóloga. Fundación Cardioinfantil, Santa Fe de Bogotá. \\ 5 Neumólogo y Director del Departamento de Investigación Fundación Neumológica Colombiana, Santa Fe de Bogotá.
}

Fecha de Recepción: 29/11/2015

Fecha de Evaluación: 26/5/2015

Fecha de Solicitud de Correcciones: 9/2/2016

Fecha de Aceptación: 24/2/2016

\begin{abstract}
Resumen
La tuberculosis (TB) es la causa más frecuente de muerte por un microorganismo bacteriano específico, causando 1,3 millones de muertes al año. Las alteraciones inmunológicas juegan un papel importante en su desarrollo (1-2). Actualmente las causas de inmunosupresión que favorecen el desarrollo de la (TB) son la infección por el virus del VIH, la desnutrición y el envejecimiento. La diabetes mellitus tipo $2(\mathrm{DM})$ presenta una pérdida de la inmunidad, aumentando el riesgo de infección y el desarrollo de enfermedad tuberculosa o su reactivación endógena (3).
\end{abstract}

Los macrófagos tienen un papel protagónico en este proceso debido al deterioro en la capacidad de fagocitosis, opsonización, producción anómala de peróxido de hidrógeno $(\mathrm{H} 2 \mathrm{O} 2)$ y mal funcionamiento de receptores $\mathrm{Fc}$ para el complemento C3; todos estos mecanismos contribuyen a la respuesta, control y destrucción del M. tuberculosis. La pérdida de estas funciones en las personas diabéticas favorece una creciente asociación con la TB que ha adquirido carácter de epidemia (4-5).

Palabras clave: Tuberculosis, Diabetes mellitus, inmunosupresión, macrófago, TB genitourinaria.
Tuberculosis and diabetes association low frequency from an outbreak recently

\begin{abstract}
Tuberculosis (TB) is the most common cause of death from a specific bacterial organism, causing 1.3 million deaths a year. Immunological disorders play an important role in its development (1-2). Currently the causes of immunosuppression favoring development (TB) are infected with HIV, malnutrition and aging. Type 2 diabetes mellitus (DM) has a loss of immunity, increasing the risk of infection and developing TB disease or endogenous reactivation (3).

Macrophages play a leading role in this process due to the deterioration in the ability of phagocytosis, opsonization, abnormal production of hydrogen peroxide $\left(\mathrm{H}_{2} \mathrm{O} 2\right)$ and malfunction of $\mathrm{Fc}$ receptors for complement $\mathrm{C} 3$; all these mechanisms contribute to the response, control and destruction of M. tuberculosis. The loss of these functions in people with diabetes favors a growing partnership with the TB epidemic has acquired character (4-5).
\end{abstract}

Keywords: Tuberculosis, Diabetes mellitus, immunosuppression, macrophage, $\mathrm{TB}$ genitourinary

\section{Introducción}

Existen varios casos publicados en la literatura desde 1879, que describen casos típicos de tuberculosis genital masculina, la cual fue descubierta 3 años más tarde por Koch. Pero sólo hasta 1980 es que realmente esta presentación cobra importancia y se produce un aumento de su incidencia en sujetos con infección VIH.

El aumento de la Diabetes mellitus tipo 2 ha generado otra población en riesgo para esta presentación, en especial aquellos con pobre control metabólico. Los diabéticos además de tener tres veces más posibilidades de infectarse y desarrollar la enfermedad tuberculosa, pueden desarrollar enfermedad extra pulmonar, entre el $15 \%$ y el $28 \%$, de las cuales la tuberculosis genitourinaria es una complicación frecuente, esta involucra próstata, vesículas seminales, conducto deferente, testículo, pene y epidídimo (6-9).

Las vías de infección aún no están claramente dilucidadas y al parecer los pulmones serían la vía de ingreso más frecuente de la M. tuberculosis. Luego de la diseminación hematógena se produce una siembra en sitios distales como en el tracto genitourinario, altamente vascularizado. Pero también se ha demostrado diseminación por medio de la vía linfática y de siembras directas (3-4-5).

Mediante este caso clínico se propone analizar la relación que existe entre el estado de inmunosupresión de los pacientes con DM y las formas extrapulmonares de la tuberculosis, haciendo énfasis en la presentación genitourinaria.

\section{Caso clínico}

Hombre de 62 años, con cuadro clínico de ocho meses de evolución de disuria, polaquiuria, masa testicular izquierda y salida de secreción blanquecina a través de la piel del escroto. Historia clínica conocida de DM tipo 2 desde hace cuatro años, sin adecuado control metabólico.

El examen físico reveló un paciente con (IMC 20,6 kg / m2), disminución de ruidos respiratorios y lesión indurada en polo superior del testículo izquierdo con salida de material blanquecino a través de orificio en piel del escroto. 
Fue admitido en la institución donde se le realizan paraclínicos: uroanálisis con presencia de 4 leucocitos por campo de gran aumento en sedimento urinario, sin aislamiento de bacterias (piuria abacteriana), hemoglobina glicosilada HbA1c: 12\% (mal control metabólico), prueba de VIH negativa, niveles plasmáticos de gonadotropina coriónica humana subunidad - $\beta$ ( $\beta$-hCG), $\alpha$-fetoproteína (AFP), deshidrogenasa láctica $(\mathrm{LDH})$ dentro de rangos normales. Se realizó una ecografía testicular, que reveló una lesión heterogénea sólida $(3,0 \times 2,8 \mathrm{~cm})$, hipoecoica, en el epidídimo izquierdo.

Paralelo a lo anterior se realizó una radiografía de tórax que mostró múltiples micronódulos de distribución al azar en ambos campos pulmonares, dichos hallazgos fueron confirmados en una imagen tomográfica de tórax.

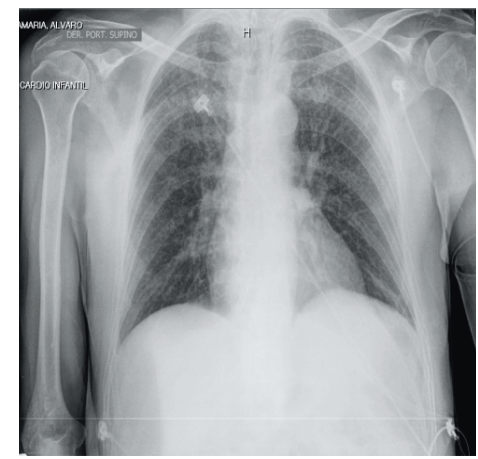

Figura 1. Radiografía de tórax PA opacidades micronódulares e intersticiales.

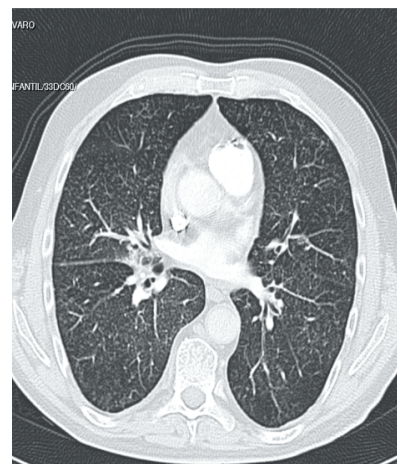

El paciente fue llevado a epididimectomía encontrándose abundante material caseoso. En el estudio histológico se observaron granulomas compuestos por células epitelioides, histiocitos, macrófagos y linfocitos, con grandes áreas de necrosis caseosa. El cultivo (Löwenstein-Jensen) fue positivo a la octava semana. Una muestra de orina fue llevada previamente a reacción en cadena de la polimerasa y GenXpert reportadas como positivas para $M$. tuberculosis y con sensibilidad a la rifampicina. Con las cuales se inicia tratamiento antituberculoso tempranamente, previo al resultado del cultivo.
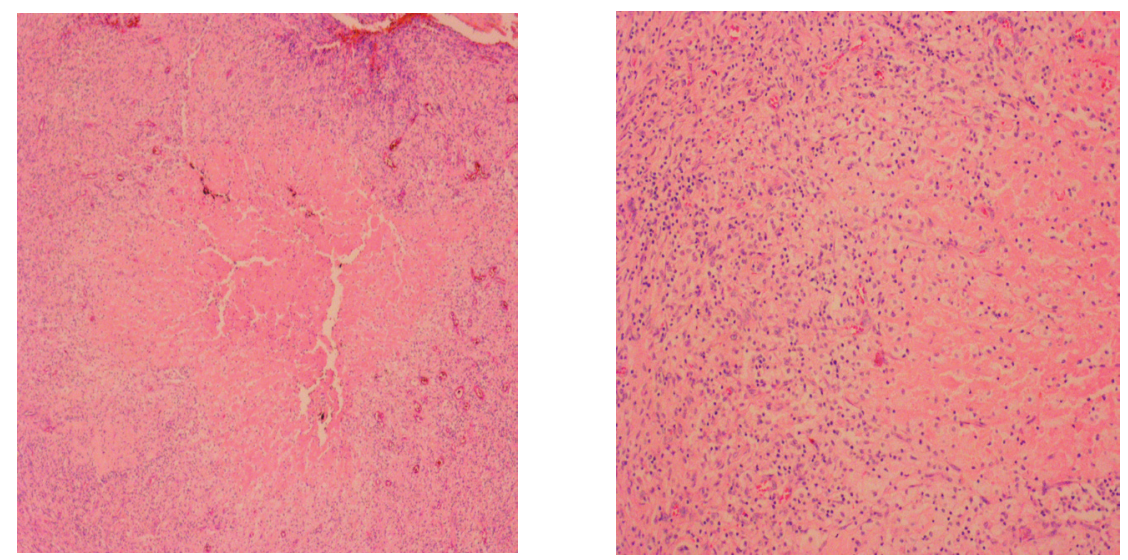

Figura 2. H\&E Granulomas con Necrosis caseosa

\section{Discusión:}

La comprensión de los mecanismos inflamatorios e inmunológicos asociados a enfermedades como la DM que influyen en la progresión de infecciones como la tuberculosis, es importante para dimensionar la severidad de la comorbilidad (10). El conocimiento puntual de algunas funciones celulares del macrófago y su papel efector en la respuesta inmune tomando como punto de partida inicial la formación del granuloma, que es un mecanismo de protección contra la difusión bacteriana, ayudan a comprender estas relaciones (11).

Posterior a la inhalación de la micobacteria, esta llega a los alvéolos donde es fagocitada por los macrófagos, los cuales la procesan en fagosomas. Estos y las células T son los principales responsables de su control y eliminación (12-13). En los sujetos con DM la regulación deficiente de la glucosa, la resistencia a la insulina y la gluconeogénesis hepática conducen a una formación excesiva circulante de ácidos grasos libres (AGL), de productos de glicosilación avanzada (PGA), que junto con un mayor nivel de estrés oxidativo, generan un estado de inflamación crónica multiorgánica (14). Esta situación conlleva al aumento de células pro-inflamatorias como CD4 + T helper tipo 1 (Th1), células (Th17) y células T citotóxicas CD8 + (CTL). Mientras que los macrófagos, las T-helper de tipo 2 (Th2) y las células T reguladoras (Treg) son suprimidas lo cual conduce a mayor destrucción tisular y al secuestro de las micobacterias dentro de compartimentos como: cavernas, abscesos y granulomas que hace más difícil su erradicación con los fármacos antituberculosos (15). Lo anterior sumado a alteraciones en las funciones como: la fagocitosis, 
la opsonización y la apoptosis que inducen a mayor número de micobacterias viables circulantes (16). En este grupo existe además una perdida en la integración de las señales celulares moleculares, deterioro en la producción del óxido nítrico sintasa y de las citoquinas inflamatorias como: TNF- $\alpha$, IL-12 e IL-18, que son indispensables para el estímulo del IFN- $\gamma$, requerido en el reclutamiento de las células NK y células T helper de tipo1 (Th1); que finalmente fortalecen la estructura del granuloma (17 - 19). Así la respuesta inflamatoria del macrófago requiere una regulación precisa para lograr el balance entre la protección y la injuria tisular (19).

La disfunción fagocítica puede ser mediada directamente a través del depósito en exceso de la glucosa y los productos de glicosilación avanzada (PGA) dentro de la célula o indirectamente a través de la acumulación de proteínas mal plegadas, que se depositan en el interior del retículo y conducen al llamado "estrés del retículo endoplásmico", fenómeno relacionado con autofagia, que conduce a degradación de componentes propios de la célula con formación de autofagosomas en lugar de la fagocitosis normal (20-21).

Estos macrófagos viajan estando infectados con micobacterias viables a través de los vasos linfáticos y sanguíneos, hasta los ganglios linfáticos regionales o en el flujo sanguíneo a cualquier parte del cuerpo. Estos pueden erosionarse, liberando los granulomas dentro del parénquima de diversos órganos (hígado, bazo, riñones, pulmones, meninges, médula ósea y otros tejidos), semejando semillas de mijo, generando el conocido patrón miliar de la infección. Por otro lado, la reactivación endógena de la tuberculosis luego de varios años, ocurre como resultado de gérmenes viables desde estos granulomas, debido al defecto inmune en el macrófago (22).

Finalmente, la TB genitourinaria representa el 5\% de los casos de tuberculosis extrapulmonar (23). Esta presentación tiene mayor incidencia en grupos de pacientes, que como los diabéticos, tienen una inmunidad comprometida, siendo una de las localizaciones más frecuentes de la TB genital la del epidídimo (24). La micobacteria tuberculosa alcanza el epidídimo por diferentes vías, una por extensión retrograda canalícular desde la próstata y las vesículas seminales y otra a través de la diseminación linfática o hematógena como puede ser la de nuestro caso a partir de una TB miliar. La latencia entre la infección pulmonar y la infección genitourinaria puede tener un periodo largo, de años antes de que aparezca la enfermedad, en algunas series se han descrito hasta 30 años. Otra fuente probable de esta condición podría ser una infección luego de la terapia con el bacilo de CalmetteGuérin práctica realizada en pacientes con cáncer de vejiga (25). El uroanálisis sugestivo se presenta con piuria abacteriana como en este caso. La ecografía es la mejor técnica para obtener imágenes del escroto, de su contenido y ayuda en el diagnóstico diferencial (26-27).

Gráfica 1. Distribución de TB extrapulmonar

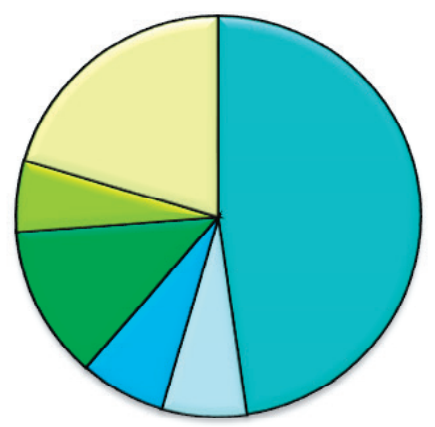

口Linfática $42 \%$

口Peritoneal $6 \%$

口Meníngea $6 \%$

aMusculoesqueletica $11 \%$

口Genitourinaria $5 \%$

口Pleural $18 \%$
Grafica 2. Distribución de TB Genitourinaria

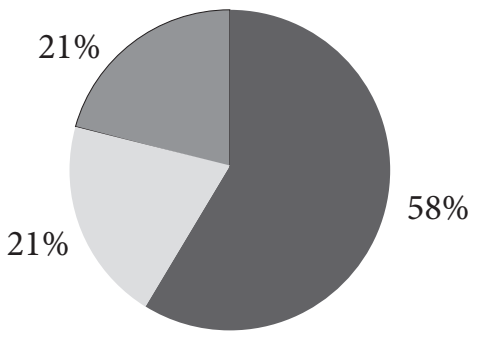

Próstata

Epidídimo

Testicular

El gold standard de la TBC genitourinaria es la identificación de la micobacteria en la orina, pero en ocasiones se requiere de material anatomopatológico para la realización del diagnóstico. Con la prueba de PCR en orina para micobacterias el diagnostico se ha facilitado. Esta es una técnica de identificación rápida con sensibilidad y especificidad de $95 \%$ y $98 \%$ respectivamente que ayuda al inicio de tratamiento temprano, pero su limitación está dada por la incapacidad para detectar si la infección es activa o es latente, lo cual hace necesario la combinación de ésta con los cultivos que determinan la actividad biológica de la micobacteria (28). Las tinciones Ziehl-Neelsen deben ser interpretadas con cuidado debido a la presencia de M. smegmatis en orina como parte de flora local normal (29), que puede conducir a falsos positivos. Todo lo anterior con el fin de realizar el diagnostico e iniciar el tratamiento rápidamente, ya que los cultivos tardan más tiempo en ser reportados. La radiografía de tórax se presenta como anormal en el $75 \%$ de los casos por ello es importante su realización (30). Se ha recomendado el tratamiento acortado supervisado. En este caso, como el paciente es diabético debe recibir 56 dosis en primera fase (dosis diaria) y 98 dosis en segunda fase (tres veces por semana) semanas. Con este esquema se pueden alcanzar tasas de curación mayores al 95\% (31).

\section{Conclusión:}

La asociación entre la diabetes y la tuberculosis podría ser el próximo desafío para el control mundial de la tuberculosis. Una mejor comprensión de la relación bidireccional de las dos enfermedades es necesaria para optimizar el tratamiento y su manejo integral. En las personas que padecen DM tipo 2 puede ser apropiada la búsqueda activa y la detección temprana de TB. Por otro lado se debe crear de un modelo similar al programa de TBC-VIH que mejore el enfoque en esta población.

El paciente discutido en este caso tenía hallazgos típicos de tuberculosis genital masculina, esta forma de presentación de tuberculosis extrapulmonar debe considerarse en hombres con quejas genitourinarias y con DM tipo 2 e historia de contacto con TB. Este caso mostró una buena respuesta a la terapia farmacológica acortada supervisada durante 9 meses dada por aumento de peso, remisión de síntomas urinarios, desaparición de lesión testicular y mejor control metabólico.

\section{Conflictos de interés}

Los autores declaran no tener conflictos de interés 


\section{Referencias}

1. World Health Organization. Global Tuberculosis Report; 2013, http://apps.who.int/iris/bitstream/10665/91355/1/ 9789241564656 eng.pdf

2. Fox GJ, Menzies D. Epidemiology of tuberculosis immunology. Adv Exp Med Biol 2013;783:1-32.

3. Blanca I. Restrepo, Larry S. Schlesinger, Impact of diabetes on the natural history of tuberculosis. diabetes research and clinical practice 106 (2014) 191 - 199.

4. Hatanaka E, Monteagudo PT, Marrocos MS et al. Neutrophils and monocytes as potentially important sources of proinflammatory cytokines in diabetes. Clin Exp Immunol 2006; 146:443-7.

5. Chacon MR, Vendrell J, Miranda M et al. Different TNFalpha expression elicited by glucose in monocytes from type 2 diabetes mellitus patients. Atherosclerosis 2007; 194: 18-25.

6. Kapoor R, Ansari MS, Mandhami A, Gulia A. Clinical presentation and diagnostic approach in cases of genitor urinary tuberculosis. Indian J Urol. 2008;24:401-405.

7. Wise GJ, Marella VK. Genitourinary manifestations of tuberculosis. Urol Clin N Am. 2003;30:111-121.

8. Petersen L, Mommsen S, Pallisgaard G. Male genitourinary tuberculosis: report of 12 cases and review of the literature. Scand J Urol Nephrol. 1993;27:425-428.

9. Chung J, Kim M, Lee T, et al. Sonographic findings in tuberculosis epididymitis and epididymo-orchitis. J Clin Ultrasound. 1997;25:390-394.

10. Wang CS, Yang CJ, Chen HC et al. Impact of type 2 diabetes on manifestations and treatment outcome of pulmonary tuberculosis. Epidemiol Infect 2009; 137:203-10.

11. Dheda K, Booth $\mathrm{H}$, Huggett JF et al. Lung remodeling in pulmonary tuberculosis. J Infect Dis 2005; 192:1201-9.

12. Cotran RS, Kumar V, Stanley L, Robbins WB. Robbins' Pathologic Basis of Disease. 5th ed. Philadelphia: WB Saunders Co; 1994.

13. D. Warren, J.R. Johnson, C.W. Johnson, C. Franklin Lowe: Genitourinary Tuberculosis Campbell's Urology (8th ed.) Saunders (2002)

14. Bertola A, Ciucci T, Rousseau D et al. Identification of adipose tissue dendritic cells correlated with obesity-associated insulinresistance and inducing Th17 responses in mice and patients. Diabetes 2012; 61:2238-47.

15. Jeong YH, Jeon BY, Gu SH et al. Differentiation of antigen-specific $\mathrm{T}$ cells with limited functional capacity during Mycobacterium tuberculosis infection. Infect Immun 2014; 82:132-9.

16. Sawant KV, McMurray DN. Guinea pig neutrophils infected with Mycobacterium tuberculosis produce cytokines which activate alveolar macrophages in noncontact cultures. Infect Immun 2007; 75:1870-7

17. Flynn JL, Chan J, Triebold KJ et al. An essential role for interferon gamma in resistance to Mycobacterium tuberculosis infection. J Exp Med 1993; 178:2249-54.

18. Flynn JL, Goldstein MM, Chan J et al. Tumor necrosis factoralpha is required in the protective immune response against Mycobacterium tuberculosis in mice. Immunity 1995; 2:561-72. 84

19. Solovic I, Sester M, Gomez-Reino JJ et al. The risk of tuberculosis related to tumour necrosis factor antagonist therapies: a TBNET consensus statement. Eur Respir J 2010; 36:1185-206.

20. Basha B, Samuel SM, Triggle CR et al. Endothelial dysfunction in diabetes mellitus: possible involvement of endoplasmic reticulum stress? Exp Diabetes Res 2012; 2012:481840
21. Zhang K, Kaufman RJ. From endoplasmic-reticulum stress to the inflammatory response. Nature 2008; 454:455-62.

22. Coats J. A Manual of Pathology. Charleston: Nabu Press; 2010.

23. GJ Wise, VK Marella. Genitourinary manifestation of tuberculosis.Urol Clin North Am. 2003;30:111-21.

24. GJ Wise, A Shteynshlyuger. An update on lower urinary tract tuberculosis. Curr Urol Rep. 2008;9:305-13.

25. BS Viswaroop, N Kekre, G Gopalakrishnan. Isolated tuberculous epididymitis: A review of forty cases. . J Postgrad Med. 2005;51:109-11.

26. Drudi FM, Laghi A, Iannicelli E, et al. Tubercular epididymitis and orchitis: US patterns. Eur Radiol. 1997;7:1076-1078.

27. Kim SH, Pollack HM, Cho KS, et al. Tuberculous epididymitis and epididymo-orchitis: sonographic findings. J Urol. 1993;150:8184.

28. A. Missirliu, D. Gasman, B. Vogt, J.D. Poveda, C.C. Abbou, D. Chopin. Genitourinary tuberculosis: rapid diagnosis using the polymerase chain reaction. Eur Urol., 30 (1996), pp. 523-524

29. S. Lenk, J. Schroeder, Genitourinary tuberculosis. Curr Opin Urol., 11 (2001), pp. 93-98

30. W.I. Christensen, Genitourinary tuberculosis: review of 102 cases Medicine (Baltimore), 53 (5) (1974), pp. 377-390.

31. N. Buchholz, R. Salahuddin, R. Haque Genitourinary tuberculosis: a profile of 55 in-patients. J Pak Med Assoc, 50 (2000), pp. 265-269 